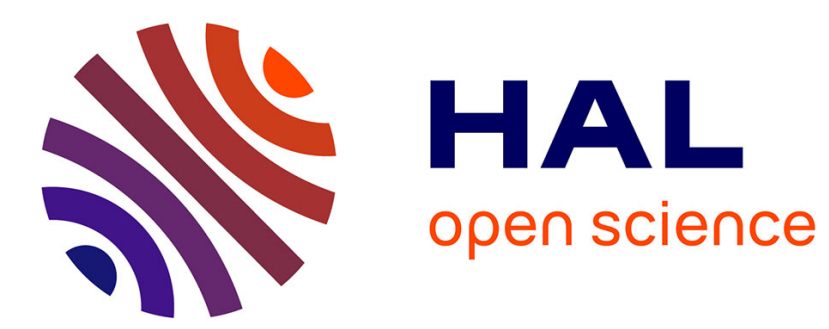

\title{
Les objectifs et les critères de sélection. La production de fibres textiles chez la chèvre, le lapin et le mouton
}

Daniel D. Allain

\section{To cite this version:}

Daniel D. Allain. Les objectifs et les critères de sélection. La production de fibres textiles chez la chèvre, le lapin et le mouton. Productions Animales, 1992, 1992, pp.161-165. hal-02711228

\section{HAL Id: hal-02711228 \\ https://hal.inrae.fr/hal-02711228}

Submitted on 1 Jun 2020

HAL is a multi-disciplinary open access archive for the deposit and dissemination of scientific research documents, whether they are published or not. The documents may come from teaching and research institutions in France or abroad, or from public or private research centers.
L'archive ouverte pluridisciplinaire HAL, est destinée au dépôt et à la diffusion de documents scientifiques de niveau recherche, publiés ou non, émanant des établissements d'enseignement et de recherche français ou étrangers, des laboratoires publics ou privés. 
D. ALLAIN et R.-G. THÉBAULT *

INRA Station d'Amélioration Génétique des

Animaux BP 2731326 Castanet-Tolosan Cedex

* INRA Domaine du Magneraud BP 52

17700 Surgères
Les objectifs et les critères de sélection

\section{La production de fibres textiles chez la chèvre, le lapin et le mouton}

Résumé. Cet article présente l'état actuel de la production de fibres textiles animales en France : le mohair produit par la chève angora, l'angora produit par le lapin angora, et la laine produite par le mouton et considérée comme un sous-produit de l'élevage ovin. Chez la chère angora, dont la toison est normalement composée d'un seul type de fibres non médullées, et chez le mouton dont la composition de la toison est très diversifiée selon les races, les paramètres zootechniques de la production de fibres sont la quantité individuelle de poils produit, la finesse et la longueur des fibres, et la pureté de la toison (rendement au lavage, taux de fibres médullées grossières, matières végétales). Chez le lapin angora, dont la toison est composée de 3 types de fibres médullées, les paramètres zootechniques de la production d'angora sont le poids de toison, la composition (poil jarreux, poil laineux) et la dureté de la toison, et la longueur des différents types de fibres. Certains critères de qualité font l'objet de mesures objectives normalisées (finesse, longueur), d'autres doivent être précisés (dureté, taux de jarres). Chez la chèvre angora et le lapin angora, la mise en place du contrôle de performances en ferme avec mesures de critères de qualité des toisons et la conduite de travaux expérimentaux en station permettent d'appréhender les composantes et les facteurs de variations de la production et de créer des génotypes particuliers dans le cadre d'une collaboration avec les éleveurs allant du simple appui technique au programme collectif d'amélioration génétique.

Les fibres textiles animales sont la laine produite par le mouton et les fibres spéciales, à savoir le mohair produit par la chèvre angora, l'angora produit par le lapin angora, le cachemire produit par certaines races de chèvres, l'alpaga et le poil de lama produit par les camélidés sud-américains. Ces appellations désignent, selon la terminologie textile, l'ensemble des poils de la toison de ces animaux, à l'exception toutefois du cachemire qui désigne exclusivement le sous-poil ou duvet fin (diamètre moyen des fibres inférieur à $18 \mu \mathrm{m}$ ) de la chèvre.

La production mondiale de fibres animales est de l'ordre de 3 millions de tonnes par an (laines : 2,9 millions $t$ en suint, mohair : $25000 \mathrm{t}$, angora : $8000 \mathrm{t}$, cachemire : $4000 \mathrm{t}$, alpaga et lama : $4000 \mathrm{t}$, ...), soit 5 à $6 \%$ de l'ensemble des fibres textiles. La production française (laines en suint : $15000 \mathrm{t}$; mohair : $22 \mathrm{t}$; angora : $150 \mathrm{t}$ ) ne couvre qu'une faible part de nos besoins industriels et/ou de consommation (laine : $120000 \mathrm{t}$; mohair : $2000 \mathrm{t}$; angora : $250 \mathrm{t}$ ). Ces fibres animales, et en particulier les fibres spéciales sont réservées à la fabrication des produits hauts de gamme.

Hormis la laine, considérée en France comme un sous produit de l'élevage ovin, mais qu'il ne faudrait plus négliger, les éleveurs de chèvre angora et de lapin angora retirent leur principale source de revenu respectivement de la production de mohair et d'angora. Ces productions sont le plus souvent une source d'originalité, de créativité et de diversité de notre agriculture : utilisation et gestion de l'espace rural défavorisé avec maintien d'un tissu économique, diversification de la production agricole, transformation du produit brut et valorisation par les éleveurs d'un produit fini, personnalisé et de haut de gamme fabriqué en petites séries.

\section{1 / La production de mohair par la chèvre angora}

\section{1 / L'élevage de la chèvre angora}

L'implantation de cet élevage en France est relativement récente, vers 1980 , avec l'importation de reproducteurs de qualité en provenance principalement du Texas mais aussi d'Australie. On dénombre aujourd'hui plus de 6000 animaux et 150 élevages répartis sur l'ensemble du territoire.

La chèvre angora est un animal relativement léger, 60 à $65 \mathrm{~kg}$ pour le bouc et 35 à $45 \mathrm{~kg}$ pour la femelle. Les naissances ont lieu au printemps, de février à avril-mai. La 1ère tonte a lieu vers l'âge de 6 mois puis 2 fois par an, en février-mars et en aoûtseptembre. La production annuelle de mohair brut est de l'ordre de $6 \mathrm{~kg}$ chez le mâle et 4,5 $\mathrm{kg}$ chez la chèvre. Contrairement aux grands pays producteurs 
(Afrique du Sud : $50 \%$ de la production mondiale ; Texas, Turquie, Argentine et Australie) où l'élevage est l'apanage des contrées arides et sèches, le mode de conduite en France est beaucoup moins extensif, avec l'utilisation d'aliments complémentaires lors de la phase de reproduction-lactation et durant la croissance des jeunes. Cela se traduit par un développement corporel plus important, de meilleures performances de reproduction et une production de mohair améliorée.

\section{2 / Caractéristiques de la toison de la chèvre angora}

La toison de la chèvre angora est normalement composée d'un seul type de fibre, le mohair, une fibre kératinique pure ayant une finesse moyenne de 25 à $40 \mu \mathrm{m}$ et une longueur de 8 à $12 \mathrm{~cm}$ après 6 mois de pousse. On peut rencontrer également jusqu'à $5 \%$ de fibres médullées indésirables. La croissance du mohair est dite permanente avec toutefois de fortes variations saisonnières de la vitesse de croissance, maximum en été et minimum en hiver. Le caractère angora, qui se traduit par un accroissement de la longueur des fibres, est additif et polygénique chez la chèvre angora.

La qualité du mohair se détermine selon sa finesse : moins de $30 \mu \mathrm{m}$ pour l'appellation "kid", de 30 à $34 \mu \mathrm{m}$ pour le "young goat" et plus de $34 \mu \mathrm{m}$ pour l"adult". La notion de longueur des fibres a un effet dépressif sur la qualité si un seuil minimum de 7 à $8 \mathrm{~cm}$ n'est pas atteint. Par contre, la présence de matériaux indésirables tels que les jarres et les autres fibres médullées, les fibres colorées, les matières végétales, urine ou fêces diminue la qualité du mohair, son rendement en lavé à fond, son utilisation et donc sa valeur marchande.

Le mohair le plus pur et le plus fin est réservé à la fabrication des produits de luxe, type tissu d'ameublement ou vêtement masculin, voire vêtement féminin et fil à tricoter, bien que ces 2 types de fabrication, très sensibles aux phénomènes de mode, permettent de valoriser des mohairs de qualité moyenne. Les mohairs grossiers sont réservés à la fabrication de couvertures.

\section{3 / Mesures des caractères de production}

Le poids de toison brut se mesure simplement lors de la tonte, mais le rendement au lavage, la finesse et la proportion de fibres médullées sont mesurées au laboratoire à partir d'un échantillon de toison.

Le rendement au lavage (entre 70 et $95 \%$ ) est mesuré après lavage en phase aqueuse.

La finesse est déterminée soit par la méthode de l'air-flow qui, selon une règle d'équivalence, mesure la perte de charge d'un flux d'air traversant une masse connue de fibres contenue dans un volume donné, soit à l'aide d'un microscope à projection. Cette dernière méthode permet également de mesurer le taux de fibres médullées.

Les techniques d'analyses d'images que nous développons et utilisons en collaboration avec l'Institut Textile de France, simplifient beaucoup ce travail et doivent nous permettre de définir une méthodologie simple, objective et peu coûteuse, de mesures des critères de qualité de la toison de la chèvre angora.
Le contrôle de performances de la chèvre angora que nous mettons en place en collaboration avec les éleveurs comprend

- un contrôle de filiation,

- l'enregistrement à la ferme, par l'éleveur, des dates et poids de tonte et du poids de l'animal,

- un pointage, par une commission, des caractéristiques de la toison de l'animal à l'âge de 15-18 mois, comprenant:

- une mesure de la longueur de mèche à l'épaule,

- une appréciation de la forme de la mèche (plate, vrillée ou crawlée), de l'homogénéité des mèches et de la couverture, et,

- une appréciation visuelle du taux de jarres,

- des analyses en laboratoires d'un échantillon de toison prélevé par la commission de pointage où sont mesurés le rendement au lavage, la finesse moyenne et le taux de fibres médullées.

\section{4 / Facteurs de variations de la production de mohair}

Les facteurs de variations non génétiques de la production de mohair sont :

- l'âge : les jeunes produisent un mohair très fin en plus faible quantité,

- le sexe : production plus grossière et plus abondante chez le mâle,

- la saison : la production de mohair est plus élevée en été,

- la conduite d'élevage incluant le niveau d'alimentation, dont les effets sont positifs sur la quantité et négatifs sur le diamètre, et le stade physiologique tel que la reproduction qui limite la production,

- les conditions de naissance, d'élevage et d'âge de la mère en ce qui concerne les 2 premières tontes exclusivement.

Les paramètres génétiques, bien que déterminés sur de petites populations et dans des conditions d'élevage extensifs, font état d'une héritabilité moyenne à forte tant pour les variables quantitatives que qualitatives (poids de toison: 0,2 à 0,4 ; finesse : 0,2 à 0,3 ; taux de jarre : 0,1 à 0,3 ) avec toutefois des corrélations génétiques positives entre le diamètre du mohair et le poids de toison, limitant ainsi toute espérance de progrès génétique rapide sur ces 2 caractères importants que sont la finesse et le poids de toison.

Outre les caractères de reproduction qui déterminent le nombre de jeunes animaux produisant le mohair le plus fin, et le format général de l'animal, le poids de toison propre, la finesse du mohair et le taux de fibres médullées sont les principaux caractères économiquement intéressants de la toison que l'on mesure.

\section{5 / Activités de recherche chez les éleveurs}

Avec la collaboration des éleveurs, qui mettent leurs animaux à notre disposition, nous étudions de façon plus précise les facteurs de variations de la production de mohair dans les conditions de l'élevage en France. L'un de nos objectifs est de mieux appréhender les composantes primaires de la production de mohair : diamètre des fibres, densité de follicules pileux, croissance des fibres selon l'incidence de la saison, de la conduite d'élevage et en fonction de l'âge de l'animal. L'acquisition de ces références doit nous 
permettre d'affiner le contrôle de performances, la qualification des reproducteurs et d'élaborer un schéma de sélection.

\section{2 / La production d'angora par le lapin angora}

\section{1 / L'élevage du lapin angora}

L'exploitation du lapin angora (Oryctolagus cuniculus angorensis) pour la production de fibres remonte au 17 ème siècle. La production de l'angora est passée de 400 t à $8000 \mathrm{t}$ depuis 1960 .

L'élevage du lapin angora se pratique sur litière de paille dans des clapiers individuels en ciment, placés sous abri sans courants d'air, en semi plein air.

La périodicité des récoltes du poil (tous les 100 jours environ) rythme l'élevage du lapin angora. Elle doit être respectée, non seulement pour la quantité et la qualité du poil récolté, mais aussi pour le maintien en bonne santé de l'animal. Afin de respecter les mues et la feutrabilité du poil fin des jeunes, la lère récolte a lieu à l'âge de 8 semaines, la 2ème, 90 jours plus tard, puis toutes les 14 semaines. La récolte du poil se pratique par dépilation, c'est à dire par épilation après traitement dépilatoire ; cette opération reste cependant coûteuse en main d'oeuvre : 20 à 30 minutes par récolte.

C'est un élevage de lapines adultes que l'on conserve le plus longtemps possible. La reproduction n'a pour but que le maintien des effectifs (taux de renouvellement : $33 \%$ ), et ne concerne qu'un nombre réduit de femelles, à savoir celles ayant les meilleurs performances poilières connues après la 4ème ou la 5ème récolte. La mise à la reproduction est donc tardive (plus d'1 an), conjuguée avec un rythme très extensif en raison de l'impossibilité de réaliser plus d'1 cycle de gestation-lactation entre 2 récoltes, et une forte saisonnalité.

\section{2 / Caractéristiques de la toison du lapin angora}

Par rapport au lapin commun, le lapin angora ne se différencie que par la longueur de son pelage, dû exclusivement à une prolongation de la durée de fonctionnement des follicules pileux : 12 à 20 semaines au lieu de 5 . Ce caractère est monogénique, récessif et autosomal.

La toison du lapin angora est hétérotypique et composée exclusivement de fibres médullées. On y retrouve des poils de garde, les jarres, raides, longs et grossiers, et un sous-poil, les duvets, plus courts, très fins frisés et environ 50 fois plus nombreux que les jarres. Le système de récolte par épilation provoque un synchronisme dans la repousse des poils. Tous les poils ont, lors de la récolte, la même durée de croissance, mais des longueurs différentes (vitesse de croissance plus rapide des jarres), soit respectivement $10 \mathrm{~cm}$ et $6,5 \mathrm{~cm}$ pour les jarres et les duvets après 14 semaines de pousse. Le diamètre des duvets est faible, de l'ordre de $15 \mu \mathrm{m}$ avec peu de variabilité, contrairement aux jarres plus grossiers, environ 50 $\mu \mathrm{m}$, et très variable, de 30 à $80 \mu \mathrm{m}$.
Les principales caractéristiques de l'angora sont: - sa finesse, égale aux meilleurs cachemires : 14-16 $\mu \mathrm{m}$,

- sa légèreté et sa capacité d'isolation thermique (fibres médullées),

- sa grande douceur au toucher, liée au faible relief des écailles de la cuticule du poil,

- sa pureté en raison de l'absence de sécrétion cutanées et des conditions d'élevage.

L'angora est utilisé presque exclusivement dans la chaîne cardée, soit en mercerie (laines fleuffées pour le tricotage), soit en bonneterie (jerseys, sous-vêtements). Ces 2 utilisations ont conduit à la sélection de 2 souches de lapins angora. L'une, la souche allemande, destinée à produire une toison laineuse, fine, avec un minimum de jarres pour faire un fil de bonneterie, est élevée principalement en Chine, en Amérique du sud et en Europe centrale. L'autre, la souche française, destinée à produire un angora jarreux, bien adaptée à la confection de laines fleuffées de haut de gamme, est principalement élevée en France ( 1200 éleveurs, 200000 lapins, 150 t).

En ce qui concerne la souche française, si la production pondérale reste un critère économiquement important, la qualité, et en particulier la jarrosité, si recherchée pour donner la laine fleuffée, est devenu le critère déterminant de la production d'angora.

\section{3 / Mesures des caractères de production}

La production moyenne d'une lapine angora est de l'ordre de $1 \mathrm{~kg} / \mathrm{an}$. Les meilleurs sujets atteignent 1,4 $\mathrm{kg}$. Tout le poil récolté sur un sujet n'a pas la même qualité. La classification française distingue 5 qualités, triées et pesées à chaque récolte du poil :

- le ler choix A (propre, non feutré, long et jarreux),

- le ler choix B (propre, non feutré, long et laineux),

- le 2ème choix (propre, non feutré et court),

- le feutré propre

- le poil souillé.

Par ailleurs le 1er choix A fait l'objet de 3 appréciations supplémentaires :

- l'homogénéité : rapport du poids de 1er choix A au poids total de la toison,

- la structure : rapport de longueur entre les jarres et les duvets dans la mèche,

- la dureté, appréciée subjectivement.

Ce dernier critère qui traduit la qualité jarreuse d'une toison exigée par les utilisateurs du secteur aval reste difficile à cerner et à mesurer objectivement. Le développement des techniques par analyses d'images nous permet de caractériser plus objectivement la toison du lapin angora en mesurant et en caractérisant le diamètre moyen, le taux et la section des jarres de la toison, avec mise au point d'une normalisation internationale des qualités d'angora brut.

Le contrôle de performances actuellement en vigueur est composé d'un enregistrement généalogique et d'un pointage des animaux avant la 4 ème récolte par un juge de l'Angora Rabbit Book de France. Ce pointage est basé sur la conformation et le développement corporel, le rendement poilier et la qualité du pelage apprécié par son homogénéité, sa structure et sa dureté. Toutefois, avec la collaboration des éleveurs, un enregistrement plus complet des performances va se mettre en place. 


\section{4 / Facteurs de variations de la production d'angora}

Les facteurs de variations non génétiques de la production d'angora sont:

- le procédé de récolte, avec un avantage à l'épilation pour la souche française,

- l'intervalle entre récoltes,

- le numéro de récolte, avec un accroissement jusqu'à la 5ème récolte et un optimum à la 7 ème récolte,

- le sexe : la production des femelles est supérieure de $19 \%$ à celle des mâles,

- la saison de récolte, avec un écart de production hiver-été de 10 à $20 \%$,

- la mise à la reproduction des femelles, qui pénalise leur niveau de production.

Les paramètres génétiques sont mals connus. Les diverses estimations font état d'une héritabilité de la production pondérale de 0,2 à 0,3 . Par contre les différents critères de qualité de la toison, en particulier ceux qualifiant la jarrosité, si recherchée dans la souche française, ne sont pas connus.

\section{5 / Activités de recherche sur l'élevage expérimental}

Dans le cadre du programme d'amélioration génétique du troupeau français de lapins angora, réalisé en collaboration avec le Syndicat National Angora Qualité, l'élevage expérimental du Magneraud (INRA) a permis de définir les caractères à sélectionner, la faisabilité de leurs mesures et d'étudier les facteurs de variations non génétiques. En outre différents protocoles sont conduits sur ces animaux :

- étude de quelques facteurs de variations de la reproduction : saison de naissance et de reproduction, âge de mise à la reproduction, saillie naturelle ou IA,

- étude des besoins nutritionnels (collaboration INRA), - création d'une souche de lapins, dite "J+", produisant une toison très dure,

- expérimentations sur les produits dépilatoires.

Par ailleurs, et bien qu'il ne s'agisse pas de fibres textiles, des études se poursuivent sur la production d'une nouvelle fourrure : l'Orylag, en application de l'étude du gène rex et des effets du photopériodisme sur les pelages. Un programme d'amélioration génétique sur 3 souches de lapins porteurs du gène rex est conduit pour améliorer les performances de reproduction et la qualité des fourrures.

\section{3 / La production de laine par le mouton}

La production de laine est très hétérogène en raison d'une grande diversité des races de mouton élevées dans notre pays. Cette diversité des toisons s'apprécie selon la présence des différents types de poils pouvant composer la toison du mouton:

- la laine sensu stricto : pas de moelle, croissance permanente, diamètre moyen de 18 à $30 \mu \mathrm{m}$ (type Mérinos, Ile de France),

- la laine médullée : très fine moelle plus ou moins discontinue, croissance permanente, diamètre moyen de 30 à $40 \mu \mathrm{m}$ (type Texel),

- le crin : poil à croissance quasi permanente, fort diamètre $(40$ à $70 \mu \mathrm{m})$, moelle continue dont le diamètre occupe $1 / 3$ à $1 / 2$ du total (type Manech),
- le jarre : poil à croissance saisonnière, vestige des poils de garde du pelage sauvage (type Limousine).

La qualité d'une laine se détermine selon la finesse moyenne et l'homogénéité de la finesse des fibres, le rendement au lavage, la présence de fibres colorées et indésirables, ainsi que divers paramètres de résistance, compression et degré de blanc utilisés par l'industrie textile. Tous ces critères font l'objet de mesures précises et objectives en laboratoires et sont à la base de la plupart des transactions commerciales internationales.

Les conditions d'élevage et les objectifs de l'élevage ovin français orienté principalement vers la production de viande ou de lait ont conduit les éleveurs à délaisser plus ou moins la production de laine. Ainsi le niveau de productivité de nos races est relativement faible, 1 à $4 \mathrm{~kg} / \mathrm{an}$, comparativement aux races spécialisées de l'hémisphère austral, 4 à 8 voire 10 $\mathrm{kg} / \mathrm{animal} / \mathrm{an}$. Mais un certain intérêt se manifeste à nouveau :

- soit pour améliorer la production de laine, en particulier chez les éleveurs de moutons à laine merinos ou semi-fines,

- soit pour mieux adapter la couverture pileuse de l'animal aux conditions climatiques dans le cadre de l'extensification de l'élevage.

L'héritabilité moyenne voire forte des caractères lainiers (poids de toison : 0,2 à 0,4 ; finesse : 0,2 à 0,5 ), devrait permettre d'atteindre à moindre coût tout objectif de sélection visant à améliorer la production de laine ou la couverture pileuse du mouton.

A ce jour l'élevage ovin français ne fait pas l'objet d'un contrôle de performances spécifiques pour la production de laine. Quelques races font toutefois l'objet d'un pointage lainier subjectif, les races Mérinos principalement, dont il peut être tenu compte pour éliminer des animaux non conformes à un certain standard de race.

\section{Conclusion}

Nos activités scientifiques sur la production de fibres chez la chèvre angora, le lapin et le mouton concernent :

- le fonctionnement des follicules pileux qui produisent les poils en relation avec les critères requis pour la transformation du produit brut,

- la métrologie et la caractérisation des fibres textiles spéciales,

- l'étude des composantes et des facteurs de variations de la production de fibres.

Ces activités sont réalisées grâce :

- aux moyens expérimentaux du Domaine du Magneraud où nous étudions, certes le modèle production de fibres textiles (lapin angora), mais aussi le modèle fourrure (lapin Orylag porteur du gène rex), sur l'espèce lapin, bien étudiée par ailleurs, - à d'étroites collaborations avec :

- les éleveurs chez qui nous expérimentons,

- les organisations professionnelles pour le suivi technique avec transfert de connaissances, le contrôle de performances et les schémas de sélection,

- l'Institut Textile de France pour la métrologie des fibres spéciales (angora, mohair) et la défini- 
tion des critères de qualité en vue de la transformation,

- les collègues de l'INRA et des laboratoires étrangers.

L'objectif étant de maîtriser la production de fibres tant au niveau de l'animal que de la population animale et de répondre, en connaissance de cause, à une demande déjà identifiée (lapin, chèvre angora) ou potentielle (mouton, chèvre cachemire, alpaga) de la profession dans le cadre d'une diversification de la production agricole.

\section{Références bibliographiques}

Allain D., Thébault R.G., Rochambeau H. de, 1991. Bases physiologiques et génétiques de la production de laine et de poils chez les petits ruminants et le lapin. International Symposium on production of hides, skins, wool and hair: evaluation in the mediterranean basin and the midle east, 2-6 November, Adana, Turkey, Com. nº 4.1.

Nicoll G.B., Bigham M.L. Alderton M.J., 1989. Estimates of environmental effectsand genetic parameters for live weights and fleece traits of angora goats. Proc N. Z. Soc. Anim. Prod. 49, 183-189.

Rochambeau H. de, Thébault R.G., 1989. Le lapin angora: production et amélioration génétique. Inra Prod. Anim., 2, $145-154$.

Rochambeau H. de, Thébault R.G., 1990. Genetics of the rabbit for wool production. Anim. Breed. Abs., 58, 1, 1-15.

Rochambeau H. de, Thebault R.G., Grun J., 1991. Angora rabbit wool production: non-genetic factors affecting quantity and quality of wool. Anim. Prod., 1991, 52, 383393.

Rogan I.M. 1988. Genetic variation and covariation in wool characteristics related to processing performance and their economic significance. Proc 3rd World Congress on Sheep and Beef cattle breeding, Paris, vol. 2, 383 - 407.

Rougeot J., Thébault R.G. 1984. Le lapin angora, sa toison, son élevage. Les Editions du point Vétérinaire, MaisonsAlfort, France, $182 \mathrm{pp}$.

Shelton M., 1981. Fiber production in "Goat Production", C. Gall Eds., Academic Press, New York, 619 pp.

Weber-Jordan C., 1989. Amélioration génétique de la toison de la chèvre Angora. Caprigène France Eds., 15 avenue de Vendome, 41018 Blois cedex, $190 \mathrm{pp}$. 